Animal Health Research Institute

Assiut Provincial Laboratory

\title{
YERSINIOSIS IN CHICKS
}

(With One Table and 10 Figures)

\author{
By \\ HEBAT - ALLAH ABD EL HALIM MOHAMED
}

(Received at 5/12/2007)

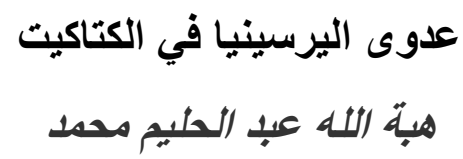

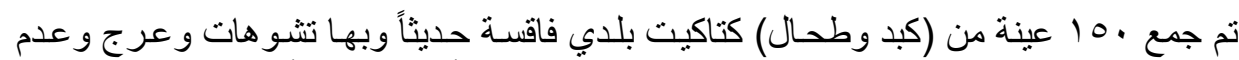

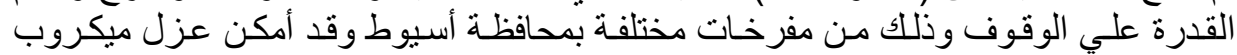

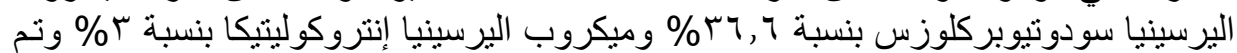

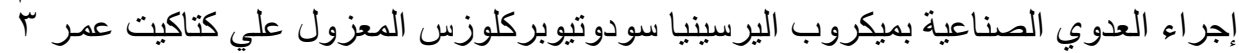

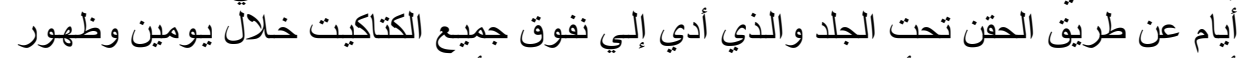

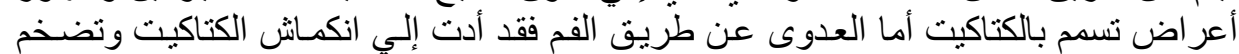

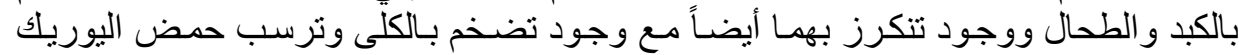

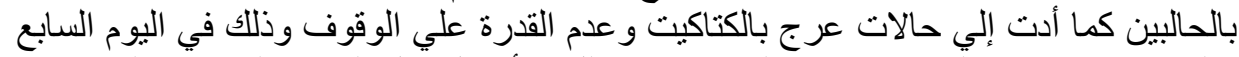

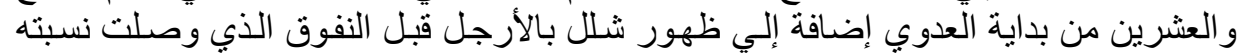

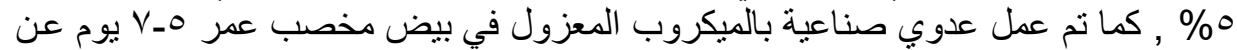

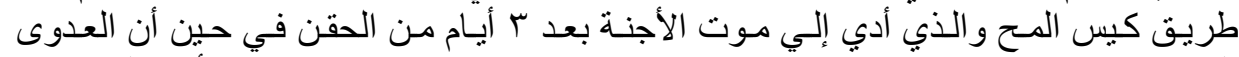

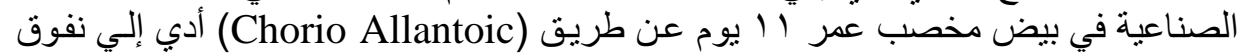

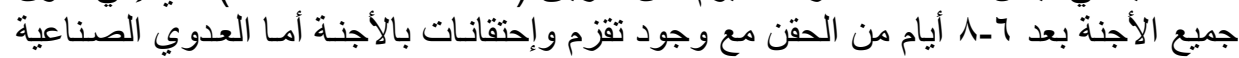

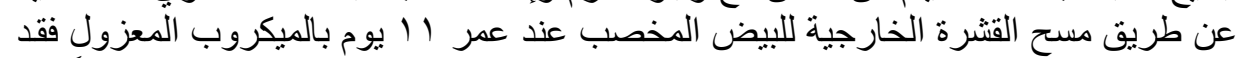

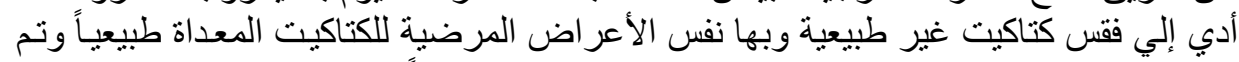

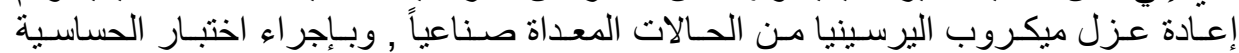

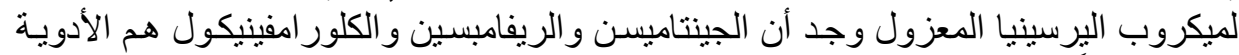
الأكثر تأثبير أ.

\section{SUMMARY}

One hundred and fifty samples from hatched chicks were collected from different hatcheries at Assiut governorate, they had abnormalities, deformity, lameness and could not stand on their legs. Yersinia pseudotuberculosis was isolated at rate of $36.6 \%$ while Yersinia enterocolitica was isolated at rate of $3 \%$. Experimental infection of 3 days old healthy chicks subcutaneous by Yersinia pseudotuberculosis isolate revealed death of all chicks within 2 days and septicemia. While 
oral route of infection revealed depression of the chicks, enlargement and congestion of liver and spleen with necrosis, enlargement of kidneys with precipitation of uric acid in the ureteres and some cases in the $27^{\text {th }}$ day postinfection had lamness and could not stand. Also paralysis of legs was appeared before death which its rate reached to $5 \%$. Inoculation of fertile chicken eggs (5 - 7 days) intra yolk sac with the isolated organism revealed deaths of all embryos within 3 days postinfection, while inoculation of fertile chicken eggs (11 days) through chorio allantoic membrane showing death of all embryos within $6-8$ days postinoculation with congestion and stunting of the embryos, on the other hand swabbing of the outershell of fertile chicken eggs with the isolated organism leads to hatching of abnormal chicks with the same symptoms of the naturally infected chicks. Reisolation of Yersinia from experimentally infected chicks was succeded. Sensitivity test revealed that gentamycin, rifampicin and chloramphenicol were the most effective drugs in vitro.

The aim of this work is designed to cover the following points:-

- Isolation and Identification of Yersinia organism and its incidence in the newly hatched chicks.

- Experimental infection of the isolated organism to 3 - days old chicks and inoculation of embryonated chicken eggs through different routes with the isolated organism.

- In vitro sensitivity test to the isolated organism to different antimicrobial discs to show the most effective drugs.

Key words: Yersiniosis, chicks, experimental infection.

\section{INTRODUCTION}

Yersinia (Y) was named after the French bacteriologist A.J.E. Yersin who first isolated the causal organism of plague in 1894. (Van Loghem 1944).

Buchanan and Gibbons (1974) Stated that Pfeiffer in (1889) was the first who described Bacillus pseudotuberculosis and he renamed it Pasteurella pseudotuberculosis in 1929. Additionally, it was called Shigella pseudotuberculosis in 1935 until Smith and Thal placed it into the genus Yersinia in 1965. Also Buchanan and Gibbons (1974) reported that the genus Yersinia was only recently adopted as a genus in the family Enterobacteriaceae to include: Yersinia pestis (the plague bacillus), Yersinia pseudotuberculosis (the pseudotubercle bacillus) and new bacillus earlier known as Pasteurella x., now designated Yersinia enterocolitica, all which cause disease in man and various animal species. 
Borst and Buitelaar (1977) recovered Yersinia pseudotuberculosis from chickens and geese.

Schiemann (1985) stated that Cefsulodin Irgasan Novobiocin CIN medium is available as $\mathrm{Y}$. selective agar medium, it requires just $18-20 \mathrm{hr}$ of incubation at $32^{\circ} \mathrm{C}$, to provide the colony.

Yersinia pseudotuberculosis $(\mathrm{Y} p)$ is the most important Pathogenic Y. species, this organism is gram - negative, forms ovoid to - coccoid rods and replicates at temperatures as low as $4^{\circ} \mathrm{C}$ in the environment. Six different serotypes are distinguished, but serotype 1 is the most commonly isolated from birds, outbreaks are common in the colder months and it is thought to have arisen from movements of rodents. Clinical signs include lethargy, dehydration, diarrhea and dyspnea. Emaciation, wasting and flaccid paresis or paralysis are common with subacute or chronic cases. Isolation of avian $\mathrm{Y}$ strains can be difficult, refrigerator enrichment for 2 weeks may improve the isolation results. (Greg et al., 1986).

Varnam and Evans (1991) and Leslie Collier et al. (1998) mentioned that Yersinias are low temperature pathogens and are able to grow at $4^{\circ} \mathrm{C}$, a distinctive feature of the genus is that many of its characteristics are temperature dependant being expressed at 28 to $30^{\circ} \mathrm{C}$, typical colonies develop as dark red surrounded by a transparent border (bull's eye). They explain this; that the Y. selective agar base have added a differential property to the medium by adding mannitol. Fermentation of mannitol result in a localized drop in $\mathrm{PH}$ causes the $\mathrm{PH}$ indicator neutral red to turn red at the center of the colony and the bile starts to precipitate giving bull's eye colony. Non fermentation of mannitol produces a colorless translucent colony.

Nagano et al., (1997) noticed that pathogenic Y.p harbour plasmid that is essential for virulence.

Palmer et al., (1998) reported that the genus Y. consists of three principal pathogenic species: $Y$ enterocolitica, $Y$ p and $Y$ pestis. Y.p and $\mathrm{Y}$ enterocolitica called non - plague yersinia and referred to simply as: (Yersiniosis). They are pathogenic for human, produce enteritis, diarrhea, mesenteric lymphadinitis and a generalized septicemia. While Y. pestis infection referred to as plague.

Nemes et al., (2000) isolated Y.p from turkey flocks and stated that it was possible to prevent spread of the disease in the flock with medication but the birds which had already shown clinical signs of the disease were incurable. 
Zurek et al., (2001) noticed that adult house flies can carry Y.p for a considerable period and therefore must be considered as a potential mechanical vector of pseudotuberculosis infection.

Keith (2002) stated that Y p. is a pathogenic gram - negative bipolar rod that has been isolated from man, mammals, rodents and domestic and wild birds. The infection of $\mathrm{Y} p$ in birds called: Avain Pseudotuberculosis. There are variations in its virulence. Its survival outside the body is similar to that of Pasteurella multocida and the clinical sings in birds are sometimes similar to those of fowl cholera, the disease may occur in acute septicemic form with symptoms like those of acute fowl cholera or death without symptoms. The oral route of infection is the natural one and the organism is shed via feces. Birds, rodents and migratory birds considered as mechanical vectors for Yp. Incubation period range from several days to two or three weeks depending on host susceptibility, strain virulence and dosage where the only affected birds are the very young birds and those whose resistance is lowered by inadequate feeding, exposure to cold or parasitic infection.

Imberechts and Die Rick (2004) reported that Yersiniosis was a zoonotic disease and caused many problems in Belgium in 2002.

\section{MATERIALS and METHODS}

\section{Materials}

Samples:

A total of 150 samples (liver - spleen) from abnormal newly hatched chicks from different hatcheries at Assiut governorate were collected.

\section{Media used:}

MacConkey's agar, blood agar, (CIN) agar, nutrient agar, triple sugar iron, Christensen's urea agar, gelatin agar, pepton broth, nitrate broth, sugar fermentation media and semisolid agar.

\section{Reagents and solutions:}

Kovac's, methyl red, hydrogen peroxide and nitrate reagent.

Stain used: Gram's stain.

\section{Experemintal birds:}

Thirty 3 - days old balady chicks and forty five embryonated chicken eggs were used in our experiment, they were obtained from the faculty of Agriculture Assiut University poultry farm.

In vitro antibiotic sensitivety discs: Include: Rifampicin $(10 \mu \mathrm{g})$, Danofloxacin $(5 \mu \mathrm{g})$ Gentamycin $(10 \mu \mathrm{g})$ Trimethoprim $(5 \mu \mathrm{g})$, Chloramphenicol $(30 \mu \mathrm{g})$ Streptomycin $(10 \mu \mathrm{g})$ Erythromycin $(15 \mu \mathrm{g})$ Ampicillin $(10 \mu \mathrm{g})$, Enrofloxacin $(5 \mu \mathrm{g})$ and Tetracycline $(30 \mu \mathrm{g})$. 


\section{Methods}

\section{1- Isolation:}

Samples from liver and spleen were taken from abnormal newly hatched chicks and cultured onto MacConkey's agar media, blood agar media and CIN agar media and incubated at $37^{\circ} \mathrm{C}$ for $24-48 \mathrm{hr}$.

\section{Identification of the isolated organism:}

The suspected colonies were examined for their morphology, and films from suspected colonies were stained by Gram's stain and biochemical reactions were done to differentiate $Y p$ from $Y$ pestis and Y. enterocolitica, according to Connie and George (1995) and Richard and Glisson (1997).

\section{Pathogenicity test:}

1- Thirty, 3 days - old healthy chicks were used and 5 birds from them were tested before experiment and proved to be free from Y. organism and the other birds were infected with Y.p and divided as follow:

$1^{\text {st }}$ group: ten, 3 days old chicks inoculated subcutaneously with $0.5 \mathrm{ml}$ of saline suspension prepared from $24 \mathrm{~h}$ old blood agar culture in a concentrate equivalent to No. 4 Mcfarland opacity tubes (according to Weisman and Singer (1976) and Soerjadi et al., (1984).

$2^{\text {nd }}$ group: ten, 3 days old chicks inoculated orally with $1 \mathrm{ml}$ of saline suspension containing $\mathrm{Y} . \mathrm{p}$ in a concentration of equivalent to No 4. Mcfarland opacity tube.

$3^{\text {rd }}$ group: Five, 3 days old chicks were left as control.

Reisolation of Y. from experimental birds were done.

2- Embryonated chicken eggs:

a- Fifteen, fertile chicken eggs (5 - 7 days) were used, 10 from them were inoculated intra yolk sac with $0.2 \mathrm{ml}$ saline containing Y. p and the other 5 left as control.

b- Fifteen, embryonated chicken eggs. (11 days) were used, 10 from them were inoculated through chorioallantoic membrane (C A M) with $0.1 \mathrm{ml}$ of saline containing Y. p and the other 5 left as control.

c- Fifteen, embryonated chicken eggs (11 days) were used, 10 from them swabed the outer egg shells of them by the saline containing Y.p and the other 5 left as control.

\section{In vitro sensitivity test:}

The determination of sensitivity of the isolated organism against different antibiotic discs was done. 


\section{RESULTS}

Bacteriological examination showed that the suspected colony was thin, dry, non haemolytic, with rough edges on blood agar plates, on MacConkey's agar plates it was clear, colorless colony, while on CIN agar the colony was dark red surrounded by a transparent border "bull's eye" (Fig.1). The organism produces heavy flocculent growth precipitated in pepton broth at $37^{\circ} \mathrm{C}$ after $24-48 \mathrm{~h}$.

Gram's stain revealed gram negative rod or coccoid, the coccoid forms usually show some bipolar staining.

Biochemical reactions revealed that the isolated organism (Yp) was catalase positive, produced urease, nitrates, ferments maltose, glucose, fructose and mannitol but could not ferment sucrose and lactose. It was negative for indole, M R, not liquefy gelatin and not produced hydrogen sulfide. On semisolid medium, Yp is motile at $25^{\circ} \mathrm{C}$ but not at $37^{\circ} \mathrm{C}$. According to the cultural character of the colonial morphology of the organisms and the biochemical reactions, the frequency of the isolated Y. was $36.6 \%$ Y.p and $3 \%$ Y. enterocolitica.

\section{Pathogenicity test:}

\section{1- Chicks:}

Chicks in group I that inoculated subcutaneously with isolated Y.p showed $100 \%$ mortality within 2 days postinoculation (P I) with signs of septicemia and congestion of all carcass (Fig. 2) and brain, (Fig. 3), congestion and enlargement of liver and spleen were present (Fig. 4). We also noticed congestion of jejunum and pancreas (Fig. 5). While chicks in group 2 which inoculated orally with Y.p isolate revealed decrease in body weight, ruffling feather, enlargement and necrosis of the liver and spleen. Also enlargement of kidneys with precipitation of urates in the ureters (Fig. 6) was found in the $27^{\text {th }}$ day PI, some birds showed lamness and could not move (Fig. 7). Paralysis of the legs (Fig. 8) was noticed in some birds before death which reached to $5 \%$.

There was no signs, lesions or death in any bird in group 3.

\section{2- Embryonated chicken eggs:}

a- chicken embryos which inoculated intra yolk sac showing death of all embyos within 3 days PI .

b- Chicken embryos which Inoculated through CAM revealed death of all embryos within $6-8$ days P I with dwarfing and congestion of all embryos (Fig. 9). 
c- While swabed the outer egg shells of chicken embyos resulted in hatching abnormal chicks (Fig. 10) and had the same signs of naturally infected newly hatched chicks.

d- All chicken embryos which left as control, hatched normal embyos.

Reisolation of $\mathrm{Y}$.p from liver and spleen of experimentally infected chicks and from allantoic fluid of inoculated chicken embryos was successful.

\section{In vitro sensitivity test:}

The effect of the different antibiotics to the Yp isolate are illustrated in Table I.

Table 1: illustrates the result of sensitivity test

\begin{tabular}{|c|c|}
\hline Antibiotic discs & Sensitivity of the Y.p isolates \\
\hline Gentamycin & +++ \\
\hline Rifampicin & +++ \\
\hline Chloramphenicol & +++ \\
\hline Ampicillin & ++ \\
\hline Tetracycline & ++ \\
\hline Trimethoprim & + \\
\hline Danofloxacin & - \\
\hline Streptomycin & - \\
\hline Erythromycin & - \\
\hline Enrofloxacin & - \\
\hline
\end{tabular}

+++ Highly Sensitive + + Moderate sensitive + + Weak sensitive . Resistant

Fig. 1: Showing bull's eye colony 


\section{DISCUSSION}

Avian pseudotuberculosis is defined as a contagious disease of domesticated and wild birds, it is characterized by an acute septicemia of short duration, followed by chronic focal infection. Y P is easily destroyed by sun light, drying, heat or ordinary disinfectant and very young birds are particularly susceptible. (Richard and Glisson 1997).

In this study the newly hatched chicks which were positive for Y. could not stand, had leg paralysis, stunting, dullness and ruffling feather.

Bacteriological examination revealed that $Y$. species was recovered from liver and spleen of infected chicks in cold months as that reported by Richard and Glisson (1997).

Experimental infection of 3 days old chicks subcutaneously with the isolated organism revealed $100 \%$ mortality, congestion of all carcass and congestion and enlargement of liver and spleen, this result is similar to that observed by Richard and Glisson (1997) and Keith (2002), but in our experiment we noticed also congestion of brain, jejunum and pancreas. The authors discussed that in susceptible bird, the organism gains entrance to the blood stream through breaks in the skin or through the mucous membranes perhaps mostly in the digestive tract, thus a bacteremia is established. Usually the bacteremic condition is of short duration but the bacteria are not all destroyes, some establish foci of infection in one or more organs such as liver, spleen, lungs or intestine giving lesions.

Experimental infection of chicks orally with isolated Y.p showed in chronic stage, decrease in body weight, ruffling feather, necrosis of liver and spleen, lamness and paralysis of legs, this result is in agreement with that observed by Jordan and Pattison (1996), Richard and Gilsson (1997) and Keith (2002) while in our experiment we also noticed enlargement, congestion of kidneys and precipetation of urates in the ureters.

Newly hatched chicks which resulted from swabed outer shells of embryonated chicken eggs showed abnormalities as shown in naturally infected newly hatched chicks. We could reisolate Yp from experimentally infected chicks and this is agreement with Weisman and Singer (1976).

Sensitivity test revealed that gentamycin, rifampicin and chloramphenicol were the most effective drugs against the isolated Y, our result is some-what similar to that observed by Palmer et al. (1998) 
who found that ampicillin, chloramphenicol and tetracycline were the most effective drugs.

Our conclusion in this study proved that $Y$. organism is active in winter months, it is easily destroyed by sunlight and ordinary disinfectants, but the broad host range of this organism suggests its wide distribution in nature and it has a proven ability to precipitate epidemics in wild birds and rodent populations. So Y.p have economic importance because it causes condemination of the carcasses due to septicemic lesions or nodules and causing decrease in the hatchability rate of newly hatched chicks. Hygenic measures and good management procedures must be used to prevent infection.

\section{REFERENCES}

Borst, G.H.A. and Buitelaar, M. (1977): Yersinia Pseudotuberculosis in birds, Tidjschr Diergeneeskd 102: 81-5

Buchanan, R.E. and Gibbons, N.E. (1974): Bergys, Manual of Determinative Bacteriology Williams and Wilkins Comp. Bult, USA.

Connie, R.M. and George, M. (1995): Diagnostic Microbiology Part II chapter 16. P. $468-469$.

Greg, J.H.; Linda, R.H. and Sounders, W.B. (1986): Clinical Avian. Medicineand Surgery P. $447-448$.

Imberechts, H. and Dierick, K. (2004): Report on zoonotic agents in Belgium in 2002. Machelem, Belgium: Veterinary and Agrochemical Research center 65 PP. (EN) Department of Bacteriology and Immunology.

Jordan, F.T.W. and Pattison, M. (1996): Yersinia Pseudotuberculosis infection (Yersiniosis, Pseudotuberculosis) In: Poultry Diseases 4th Edition P. 46-47.

Keith, R. Gooderbam (2002): Yersinia Pseudotuberculosis infection (Yersiniosis, Pseudotuberculosis) In: Poultry Diseases $5^{\text {th }}$ Edition Jordan P. 133 - 134.

Leslie, C.; Albert, B. and Max, S. (1998): Yersinia infections other than plague in: Topley \& Wilson's Microbiology and Microbial infections. Systematic Bacteriology. Vol . 3 chapter 45 P. 905910.

Nagano, T.; Kiyohara, T.; Suzuki K.; Tsubokura, M. and Otsuki, K. (1997): Identification of pathogenic Strains within serogroups of Yersinia pseudotuberculosis and the presence of non- 
pathogenic strains isolated from animals and the environment. J. Vet. Med. Sci. Mar. 59 (3): 153-8.

Nemes, C.; Banda, T.; Glavits, R.; Cseplo, A. and Visnyei, S. (2000):

Disease in large numbers in a turkey flock caused by yersinia pseudotuberculosis. Magyar Allatorvosok lapja 122 (10) 579585.

Palmer, S.R.; Soulsby, L. and Simpson, D.I.H. (1998): Biology Clinical Practice and Public health control P. 281-286.

Pfeiffer, A. (1889): Ueber die Bacillare Pseudotuberculosis bei Nagethieren, Verlag Von Georg Thieme, Leipzig.

Richard, R.B. and Glisson, J.R. (1997): Pseudotuberculosis. In Diseases of Poultry, edited by Calnek, B.w., John Barnes, H., Beard, C.W., Mcdougald L.R. and Saif, Y.M. 10 ${ }^{\text {th }}$ edition P. $314-$ 318.

Schiemann, D.A. (1985): Yersinia enterocolitica in milk and dairy products. J. Dairy Sci, 70. 303.

Smith, J.E. and Thal, E. (1965): A taxonomic study of the genus Pasteurella using a numerical technique, Acta Path. Microbial Scand., 64: 213-23.

Soerjadi - Liem, A.S.; Snoeyebos, G.H. and weinack, O.M. (1984): Establishment and Competitive Exclusion of Yersinia enterocolitica in the gut of Monoxenic and Holoxenic chicks. Avian Diseases. Vol. 282 No. 1: 256 - 260.

Van Loghem, J.J. (1944): The classification of plague- bacillus. Antonie Van Leeu Wen hoek. J. Microbiol. Serol. 10:15.

Varnam, A.H. and Evans, M.G. (1991): Yersinia In (Foodborne Pathogens' Wolf publishing Ltd, London, England

Weisman, J. and Singer, N. (1976): Isolation of Yersinia (Pasteurella) Pseudotuberculosis from the palm Dove (Streptopelia senegalensis). Avian Diseases Vol: 20 No. I: $202-204$.

Zurek, L.; Denning, S.S.; Schai, C. and Watson, D.W. (2001): Vector Competence of Musca domestica (Diptera: Muscidae) for Yersinia Pseudotuberculosis, Journal of Medical Entomology 38 (2) $333-335$. 
Assiut Vet. Med. J. Vol. 54 No. 116 January 2008 
Fig . 2 Subcutaneously infected chicks showing congestion of all carcass .
Fig . 3 Congestion experimentally infer 
Fig . 5 Congestion and inflammation of jeujenum and pancreas of experimentally infected chick .
Fig . 6 Showing enlas kidneys with precip urates in the ureters infected chick . 
Fig. 2: Subcutaneously infected chicks showing congestion of all carcass

Fig. 3: Congestion of brain of experimentally infected chick.

Fig. 4: Subcutaneously infected chicks showing abnormal liver with congestion and petecheal haemorrhages.

Fig. 5: Congestion and inflammation of jejunum and pancrease of experimentally infected chick.

Fig. 6: Showing enlargement of kidneys with precipitation of urates in the ureters of orally infected chick.

Fig. 7: Orally infected chick showing lamness and could not move.

Fig 8: Orally infected chick showing paralysis of legs.

Fig. 9: Infected chicken embryos through $\mathrm{C}$ A $\mathrm{M}$ showing congestion and dwarfing (left), normal (right).

Fig. 10: Showing abnormal newly hatched chick after swabbed of the outer eggshell with Y.P. 
Assiut Vet. Med. J. Vol. 54 No. 116 January 2008 